\title{
Tailoring nuclear receptor activation
}

Agents that target multiple diseaserelated metabolic parameters, such as insulin sensitivity, dyslipidaemia and weight gain, could provide more effective and convenient treatment for type 2 diabetes. Writing in PNAS, Artis et al. describe how such agents targeting the peroxisome proliferatoractivated receptor (PPAR) family of nuclear receptors can be designed using a fragment-based lead-discovery strategy.

Two classes of marketed drugs already target specific members of the PPAR family, which have a key role in regulating metabolic processes and inflammation. Fibrates, which activate PPAR $\alpha$, are used as lipidlowering agents, whereas glitazones, which activate PPAR $\gamma$, have insulinsensitizing properties. Furthermore, PPAR $\delta$ activators have also been investigated as potential drugs for dyslipidaemia and weight loss.

So, compounds that can stimulate all three PPARs could target the multiple risk factors that are commonly present in patients with diabetes and reduce the need for polypharmacy and its associated risks and inconvenience. In addition, tailoring the level of receptor activation could be important in addressing the cardiovascular risk that has recently been associated with full PPAR $\gamma$ agonism.

To tackle the challenge of identifying such compounds, the authors applied a lead-discovery approach that they had previously successfully applied to the kinase and phosphodiesterase enzyme families. First, 170 low-molecular mass 'fragments' that activated one or more of the PPAR receptors, albeit weakly, were identified from a biochemical screen. Co-crystal structures of these compounds with at least one of the PPARs were obtained where possible, which led to the identification of a promising scaffold on which to build compounds that activate all three PPARs.

A computational structure-based approach was then used to guide synthesis of derivatives of this scaffold for testing in cellular assays. One such derivative, indeglitazar, which activated all three PPARs (acting as a partial rather than a full agonist of PPAR $\gamma$ ) and possessed excellent drug-like properties, was selected for development.

In rodent models of diabetes, indeglitazar had strong beneficial effects on levels of glucose, triglycerides and cholesterol. Furthermore, it led to weight loss in both rodents and primates. In this respect, compared with the full PPAR $\gamma$ agonist rosiglitazone, indeglitazar was less potent in stimulating an adiponectin response, which might be linked to the undesirable weight gain that is observed with glitazones.

On the basis of this promising therapeutic profile, indeglitazar has progressed to Phase II trials in patients with type 2 diabetes. The compounds

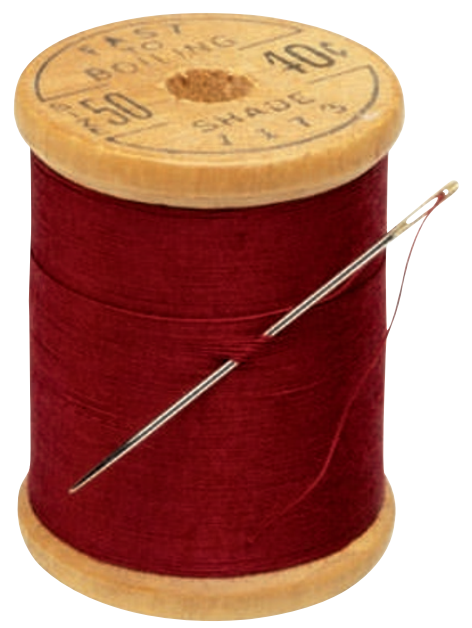

and approach described could also be valuable for future investigation of the effects of varying levels of activation of PPARs, and could provide more potential drugs for patients with metabolic disorders as subpopulations become better defined.

Peter Kirkpatrick

ORIGINAL RESEARCH PAPER Artis, D. R. et al. Scaffold-based discovery of indeglitazar, a PPAR pan-active anti-diabetic agent. Proc. Natl Acad. Sci. USA 106, 262-267 (2009)

FURTHER READING Hajduk, P. J. \& Greer, J.

A decade of fragment-based drug design: strategic advances and lessons learned. Nature Rev. Drug Discov. 6, 211-219 (2007) | Tsai, J. et al. Discovery of a selective inhibitor of oncogenic B-Raf kinase with potent antimelanoma activity. Proc. Natl Acad. Sci. USA 105, 3041-3046 (2008)| Card, G. L. et al. A family of phosphodiesterase inhibitors discovered by cocrystallography and scaffold-based drug design. Nature Biotechnol. 23, 201-207 (2005) 